\title{
The Role of Current Account Balance in Forecasting The US Equity \\ Premium: Evidence From A Quantile Predictive Regression Approach ${ }^{\#}$
}

\author{
RANGAN GUPTA ${ }^{*}$, ANANDAMAYEE MAJUMDAR ${ }^{* *}$, MARK E. WOHAR ${ }^{* * *}$
}

\begin{abstract}
The purpose of this paper is to investigate whether the current account balance can help in forecasting the quarterly S\&P500-based equity premium out-of-sample. We consider an out-of-sample period of 1970:Q3 to 2014:Q4, with a corresponding in-sample period of 1947:Q2 to 1970:Q2. We employ a quantile predictive regression model. The quantilebased approach is more informative relative to any linear model, as it investigates the ability of the current account to forecast the entire conditional distribution of the equity premium, rather than being restricted just to the conditional-mean. In addition, we employ a recursive estimation of both the conditional-mean and quantile predictive regression models over the out-of-sample period which allows for time-varying parameters in the forecast evaluation part of the sample for both these models. Our results indicate that unlike as suggested by the linear (mean-based) predictive regression model, the quantile regression model shows that the (changes in the) real current account balance contains significant outof-sample information when the stock market is performing poorly (below the quantile value of 0.3), but not when the market is in normal to bullish modes (quantile value above 0.3). This result seems to be intuitive in the sense that, when the markets are performing average to well, that is performing around the median and above of the conditional distribution of the equity premium, the excess returns is inherently a random-walk and hence, no information, from a predictor (changes in the real current account balance) is necessary.
\end{abstract}

JEL Codes: C22; C53; F32; G10

Keywords: stock markets; current account; predictability; quantile regression

\footnotetext{
\# We would like to thank two anonymous referees for many helpful comments. However, any remaining errors are solely ours.

Department of Economics, University of Pretoria, Pretoria, 0002, South Africa; E-mail address: rangan.gupta@up.ac.za.

Center for Advanced Statistics and Econometrics, Soochow University, Suzhou, China; E-mail address: anandamayee.majumdar@gmail.com.

College of Business Administration, University of Nebraska at Omaha, 6708 Pine Street, Omaha, NE 68182, USA, and School of Business and Economics, Loughborough University, Leicestershire, LE11 3TU, UK. Email: mwohar@unomaha.edu.
} 


\section{INTRODUCTION}

The existing literature on forecasting US stock returns (and or equity premium) is vast. Practitioners in finance require real-time forecasts of stock returns for asset allocation, and academics in finance are interested in stock return forecasts, since they have important implications for tests of market efficiency, which in turn, helps to produce more realistic asset pricing models (Goyal and Welch, 2008; Rapach and Zhou, 2013; Meligkotsidou et al., 2014). However, stock return forecasting is highly challenging, since it inherently contain a sizable unpredictable component. Understandably, a wide array of models (univariate and multivariate; linear and nonlinear), and predictors (domestic and international financial and macroeconomic; institutional and behavioral) have been used (see Aye et al., (forthcoming) and references cited therein for further details). Not surprisingly, the evidence is mixed.

In a seminal contribution, Mercereau (2003a) develops a dynamic general equilibrium model to show that the current account may help predict future stock market performance. The model reasoned that, because the current account is derived from the optimal portfolio and consumption or savings choices of the agents, it should simultaneously incorporate and reflect all the relevant information agents have about future stock market performance (including those not observed by econometricians). This forecasting property was formally expressed by a set of Granger causality and Granger causal priority propositions in Mercereau (2003a, b), and then tested using US data by Mercereau (2003b) to provide some evidence of current account being able to predict excess stock returns based on an in-sample exercise.

Against this backdrop, and under the widely held view that predictive models require out-of-sample (forecasting) validation (Campbell, 2008), the objective of this paper is to investigate whether the current account can help in forecasting the quarterly S\&P500- 
based equity premium. For our purpose, we consider an out-of-sample period of 1970:Q3 to 2014:Q4, with a corresponding in-sample period of 1947:Q2 to 1970:Q2, with the overall period of 1947:Q2 to 2014:Q4 covering all the eleven post-World War II recessions in the US.

Traditionally, studies dealing with stock returns and equity premium prediction have used a linear predictive regression framework (Goyal and Welch, 2008). However, recent contributions to the literature have pointed out that the relationship between returns and predictors is not linear (Bekiros and Gupta, 2015; Bekiros et al., forthcoming a, b). Given this, the literature has resorted to Markov-switching, smooth transition threshold, neural networks, non or semi-parametric, time-varying coefficient, and quantile regression models (see Rapach and Zhou, (2013); Meligkotsidou et al., (2014) for detailed literature reviews in this regard). In this paper, we address the issue of non-linearity (and nonnormality), which we show to exist, between excess returns and the predictive variable (i.e., current account), by considering a quantile predictive regression model. The quantile-based approach is more informative relative to any linear model, as it investigates the ability of the current account to forecast the entire conditional distribution of the equity premium, rather than being restricted just to the conditional-mean. Looking at just the conditional mean of the equity premium series may 'hide 'interesting characteristics as it can lead us to conclude that a predictor has poor predictive performance, while it is actually valuable for predicting certain parts of distribution of excess returns. Note that, on the one hand, unlike the Markov-switching and the smooth threshold models, we do not need to specify number of regimes of the excess returns (for instance bear and bull) in an ad hoc fashion with the quantile model. While on the other hand, the quantile approach has added advantage over the non- or semi-parametric, neural networks and time-varying approaches, as we can study 
each point of the conditional distribution characterizing the existing nature of the stock market.

In addition, business cycle fluctuations are likely to induce the slope coefficients associated with the predictor to vary across quantiles (Rapach and Zhou, 2013), to the extent that the current account may contain significant information for the lower or upper quantiles, but not for the whole, of the conditional distribution of the equity premium. The quantile predictive regression approach, which allows us to integrate this information, would thus lead to additional benefits over the standard linear and popular nonlinear methods. Note that, since the quantile regression approach studies the entire conditional distribution, which captures various states of the stock market, it has an inherent timevarying nature to the estimation process. Though, by pursuing a recursive estimation of both the conditional-mean and quantile predictive regression models over the out-of-sample period, we make both the models have time-varying parameters in the forecast evaluation part of the sample. Note that, the split between the in-sample (1947:Q2-1970:Q2) and the out-of-sample period (1970:Q3-2014:Q4) is determined statistically by using the multiple structural break test of Bai and Perron (2003), with us ensuring that all the breaks fall in the out-of-sample.

To the best of our knowledge, this is the first attempt to analyse the forecastability of the current account vis-à-vis the US equity premium, utilizing a quantile regression approach. But it must be pointed out that studies by Cenesizoglu and Timmermann (2008), Cenesizoglu and Timmermann (2012), Meligkotsidou et al., (2014) have shown that forecasting gains (relative to the benchmark model of historical average) could be obtained using quantile predictive regressions at certain parts of the conditional distribution of the US equity premium based on the standard macroeconomic and financial predictors suggested by Goyal and Welch (2008). Bekiros et al., (forthcoming) also shows this to be 
the case when one uses economic policy uncertainty as a predictor. The rest of the paper is organized as follows: section 2 presents the econometric methodology while section 3 describes the data and discusses the results. Section 4 concludes.

\section{Quantile Predictive Regression MODEL}

The classical mean regression model is given by:

$$
r_{t+1}=\alpha_{i}+\beta_{i} x_{i, t}+\varepsilon_{t+1}
$$

where $r_{t+1}$ is the observed excess return at time $t+1, x_{i, t}$ is a specific regressor / predictor at time $t$, which in our work is current account, and $\varepsilon_{t+1}$ is the error term assumed to be independent with zero mean and variance $\sigma^{2}$. The ordinary least squares (OLS) estimators $\hat{\alpha}_{i}, \hat{\beta}_{i}$ of the parameters in the predictive mean regression model can be estimated by minimizing the quadratic expected loss, $\sum_{t=0}^{T-1}\left(r_{t+1}-\alpha_{i}-\beta_{i} x_{i, t}\right)^{2}$ with respect to $\alpha_{i}, \beta_{i}$. Then, the point forecast of the equity premium at time $t+1$, can be obtained as: $\hat{r}_{i, t+1}=$ $\hat{\alpha}_{i}+\hat{\beta}_{i} x_{i, t}$

The aforementioned model specification is primarily devised to predict the mean of $r_{t+1}$, and not its entire distribution. Koenker and Bassett (1978) showed that Quantile Regression estimators are more efficient and robust than mean regression estimators in cases where nonlinearities and deviations from normality exist, with both these features existing in our data (as discussed below). Hence, we consider the quantile regression model of the following form:

$$
r_{t+1}=\alpha_{i}^{(\tau)}+\beta_{i}^{(\tau)} x_{i, t}+\varepsilon_{t+1} \quad i=1, \ldots, N,
$$


where $\tau \in(0,1)$ and $\varepsilon_{t+1}$ are assumed independent derived from an error distribution $g_{\tau}(\varepsilon)$ with the $\tau$-th quantile equal to 0 . Model (2) suggests the $\tau$-th quantile of $r_{t+1}$ given $x_{i, t}$, is $Q_{\tau}\left(r_{t+1} \mid x_{i, t}\right)=\alpha_{i}^{(\tau)}+\beta_{i}^{(\tau)} x_{i, t}$, where the intercept and the regression coefficients depend upon $\tau$. The estimators of the parameters of the linear quantile regression model in Eq. (2), $\alpha_{i}{ }^{(\tau)}, \beta_{i}{ }^{(\tau)}$, can be obtained by minimizing the sum $\sum_{t=0}^{T-1} \rho_{\tau}\left(r_{t+1}-\alpha_{i}{ }^{(\tau)}-\beta_{i}{ }^{(\tau)} x_{i, t}\right)$, where $\rho_{\tau}(u)=u(\tau-I(u<0))=\frac{1}{2}[|u|+(2 \tau-1) u]$. The forecast of the $\tau$-th quantile of the distribution of the equity premium at time $t+1$ is obtained as $\hat{r}_{i, t+1}(\tau)=\alpha_{i}^{(\tau)}+$ $\beta_{i}^{(\tau)} x_{i, t}$

\section{EMPIRICAL RESULTS}

The dataset used in the present study covers the quarterly period 1947:Q1-2014:Q4 and incorporates two variables, namely the US equity premium and the balance on current account in real terms. The equity premium is calculated as the difference of the continuously compounded CRSP value-weighted stock market returns, including dividends and the three-month Treasury bill rate. ${ }^{1}$ The equity premium, is calculated based on the data available on the website of Professor Amit Goyal at: http://www.hec.unil.ch/agoyal/.The seasonally adjusted nominal balance on the current account is deflated by the seasonally adjusted GDP deflator (as in Mercereau (2003b)) with a base year of 2009 to yield the real current account balance, with both these variables derived from FRED database of the Federal Reserve Bank of St. Louis (https://research.stlouisfed.org/fred2/). The start and end dates of the sample are purely driven by the data availability of the current account balance and the equity-premium respectively, at the time of writing this paper. Figure 1 plots the equity premium or excess returns (EXR) and the real current account balance in levels

\footnotetext{
${ }^{1}$ The CRSP Index (which includes the NYSE, AMEX, and Nasdaq) is believed to provide a better proxy for market returns than S\&P 500 Index as it is much broader than the latter (Rapach and Zhou, 2013).
} 
(RCA), and the latter also first-differences (DRCA) to ensure stationarity. ${ }^{2}$ As we lose the first observation due to differencing of the current account, our effective sample starts in 1947:Q2, giving us a total of 271 observations till 2014:Q4. As can be seen from Figure 1, the real current account balance was in general in surplus, but consistent deficit started to emerge (and has been so) since 1977:Q1, with the highest deficit recorded in 2006:Q3. First-differences in real current account are found to become very volatile since 1974:Q2, while the movements in the excess returns have been pretty similar throughout the sample period. However, there is similarity in the movements of the EXR and DRCA especially starting around the 1970s. Table 1 presents the summary statistics of EXR and DRCA, with both variables found to be non-normal based on the rejection of the null of normality under the Jarque-Bera test at the highest level of significance. Heavy-tails (negative for EXR and positive for DRCA) provide a preliminary motivation to look at quantile predictive regressions.

Following Bekiros et al., (forthcoming), to determine our in-sample and out-ofsample segmentation with the models in Eq. (1) and Eq. (2) being estimated recursively over the latter, we conduct the Bai and Perron (2003) test of multiple structural breaks on equation Eq. (1). The powerful WDmax test under the specification of 1 to $\mathrm{M}$ globally determined breaks reveal four breaks, specifically at 1970:Q3, 1974Q4, 2003Q2, and 2009Q2. Hence, our in-sample covers 1947:Q2-1970:Q2, while the rest is utilized as the out-of-sample. ${ }^{3}$ Note that, our out-of-sample period also corresponds with start of the

\footnotetext{
${ }^{2}$ Complete details of the unit root tests on the real current account balance are available upon request from the authors. Note that Mercereau (2003a, b) also found that the real current account was only stationary after firstdifferencing.

${ }^{3}$ Complete details of the structural break tests are available upon request from the authors. Note that, with both the conditional mean-based and the quantile models estimated recursively over the out-of-sample horizon which includes all the breaks, we are able to account for possible nonlinearity that would arise in the relationship between the first-differences of the real current account and the equity premium due to such regime shifts. Hence, we provide a fair comparison across the two types of model by controlling for such nonlinearity due to structural breaks, and in the process, this allows us to gauge the possibility of the importance of nonlinearity that exists inherently at various phases of the equity market with the current account balance.
} 
Figure 1: Data Plots of ExCess Stock Returns And CuRrent AcCount Balance 1A. EXCESS STOCK RETURNS:

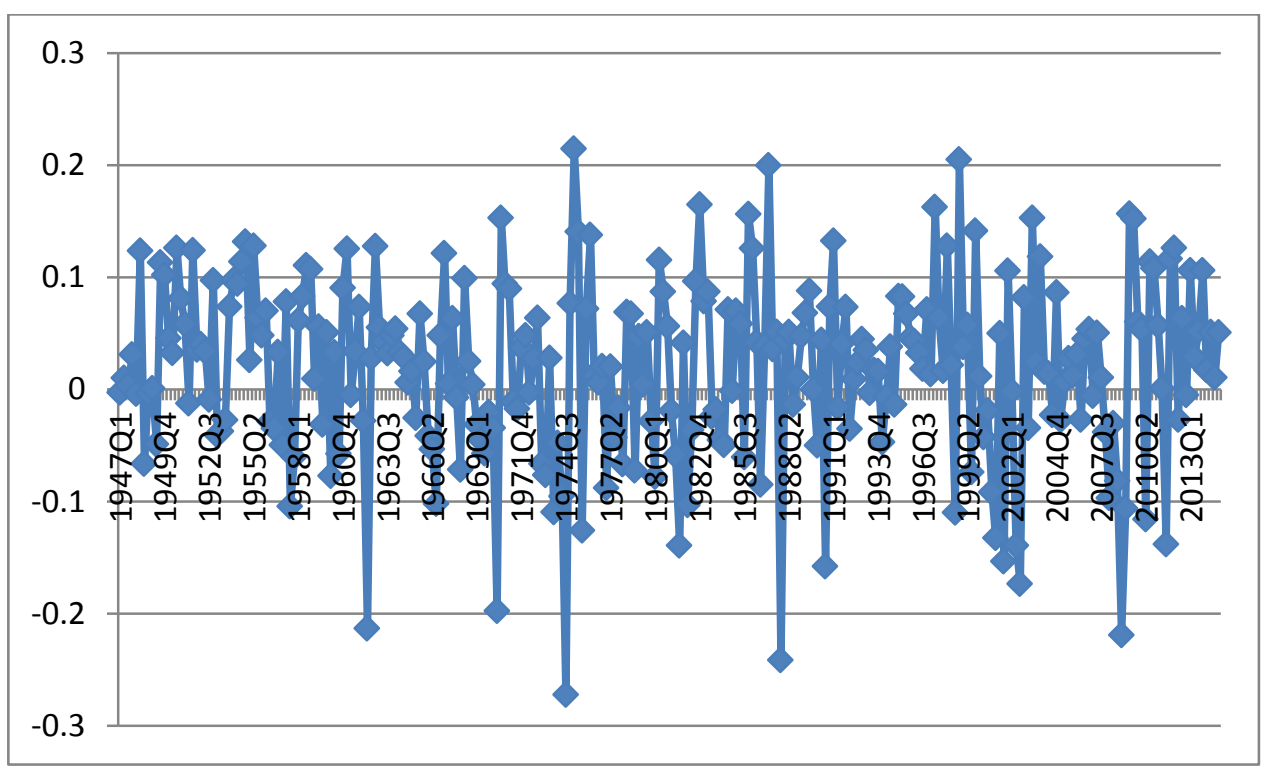

1B. REAl CURRENT ACCOUNT In LEVELS (RCA):

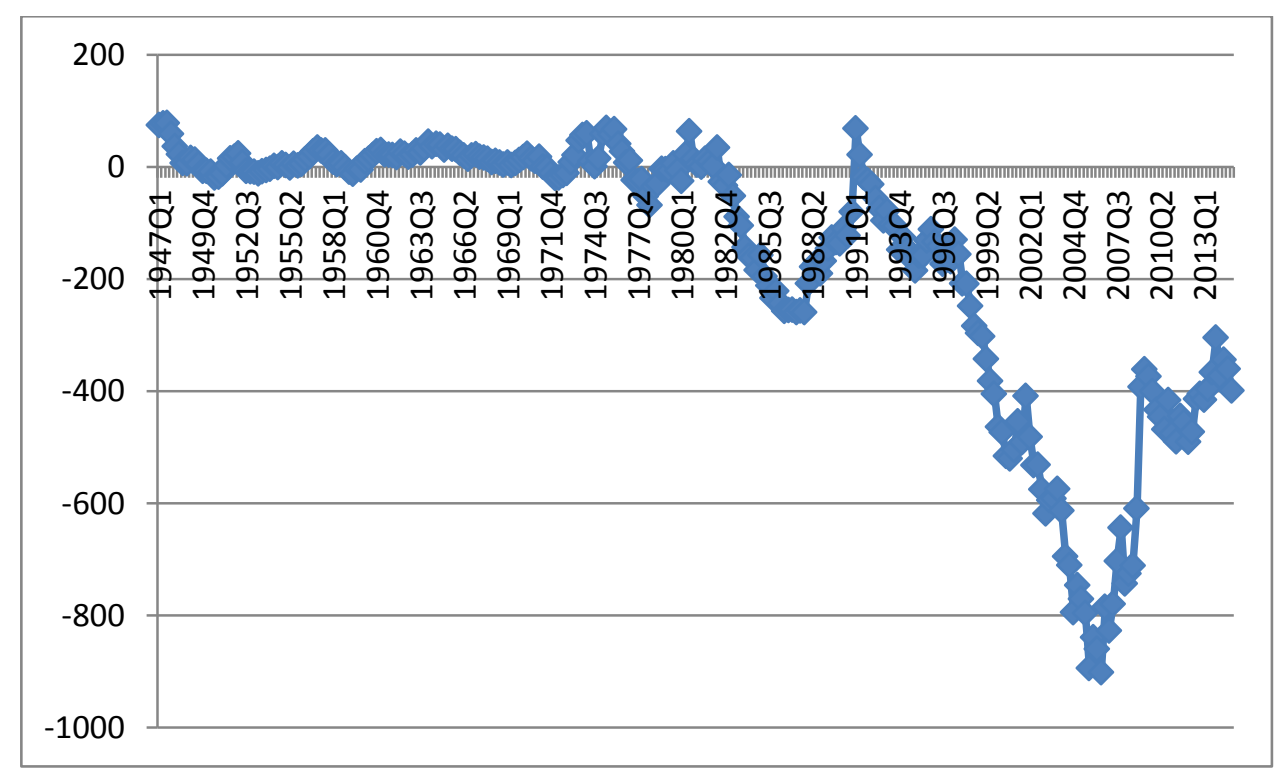


1C. REAL CURRENT ACCOUNT IN FIRST-DIFFERENCES (DRCA):

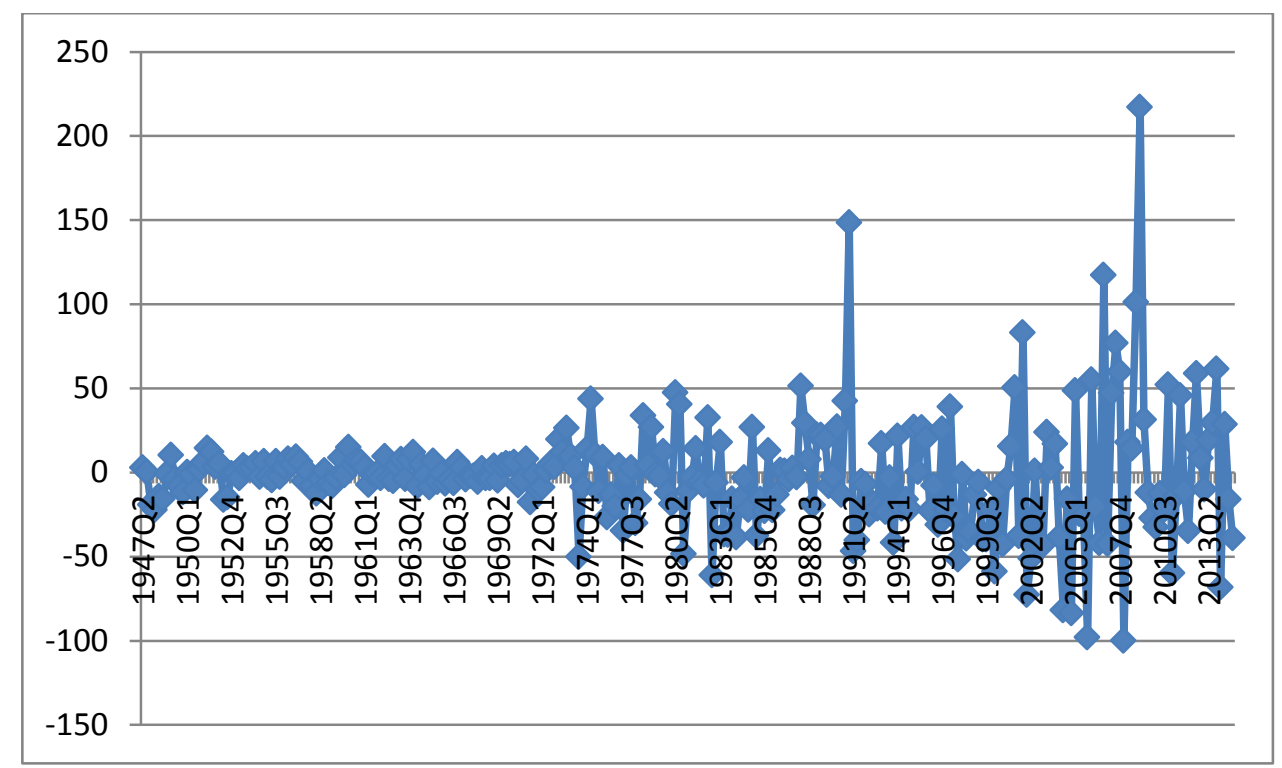

TABLE 1. SUMMARY STATISTICS

\begin{tabular}{|c|c|c|c|}
\hline STATISTIC & EXR & DRCA & $\begin{array}{c}\text { RESIDUAL } \\
\text { OF Eq. (1) }\end{array}$ \\
\hline Mean & 0.0197 & -1.7488 & 0.0000 \\
\hline Median & 0.0282 & -2.6168 & 0.0102 \\
\hline Maximum & 0.2145 & 217.0709 & 0.1925 \\
\hline Minimum & -0.2723 & -99.8806 & -0.2849 \\
\hline Std. Dev. & 0.0784 & 32.3057 & 0.0784 \\
\hline Skewness & -0.5937 & 1.5765 & -0.5893 \\
\hline Kurtosis & 3.9672 & 12.8164 & 3.9357 \\
\hline Jarque-Bera & 26.4820 & 1200.3420 & 25.4741 \\
\hline$p$-value & 0.0000 & 0.0000 & 0.0000 \\
\hline Obs. & 271 & 271 & 270 \\
\hline
\end{tabular}

Note: EXR and DRCA denotes excess stock returns and changes in real current account balance respectively; Eq. (1): $r_{t+1}=\alpha_{i}+\beta_{i} x_{i, t}+\varepsilon_{t+1}$; Std. Dev. symbolizes the Standard Deviation; $p$-value corresponds to the test of normality based on the Jarque-Bera test. 
floating exchange rate regime in the US between 1971 and 1973. Then, when we apply the Jarque-Bera test on the residuals recovered from Eq. (1) the null of normality is overwhelmingly rejected at the highest levels of significance as a result of negative skewness and excess kurtosis. This result has also been reported in Table 1. Furthermore, the Brock et al., (1996, BDS) test when applied on the residuals of Eq. (1) without and with the predictor (DRCA), also rejects the null of i.i.d. at all possible dimensions at conventional levels of significance, thus providing strong evidence of nonlinearity in the excess returns and also between the US equity premium and DRCA. ${ }^{4}$ The results from the structural instability analysis as well as the nonlinearity test, highlight, on the one hand, the inappropriateness of the linear predictive regression specification defined in Eq.(1), and on the other hand indicate the necessity to employ a quantile predictive regression, as in Eq. (2).

\section{TABLE 2. BDS TEST}

\begin{tabular}{|c|c|c|c|c|}
\hline & $\begin{array}{c}z \text {-statistic of } \\
\text { Residual } \\
\text { Benchmark Model }\end{array}$ & $p$-value & $\begin{array}{c}z \text {-statistic of } \\
\text { Residual } \\
\text { Eq. }(1)\end{array}$ & $p$-value \\
\hline 2 & 2.2694 & 0.0232 & 1.9063 & 0.0566 \\
\hline 3 & 3.4468 & 0.0006 & 3.1195 & 0.0018 \\
\hline 4 & 4.1826 & 0.0000 & 3.8346 & 0.0001 \\
\hline 5 & 4.5101 & 0.0000 & 4.2734 & 0.0000 \\
\hline 6 & 4.6836 & 0.0000 & 4.6189 & 0.0000 \\
\hline
\end{tabular}

Note: $m$ stands for the number of (embedded) dimension which embed the time series into $m$-dimensional vectors, by taking each $m$ successive points in the series; Benchmark Model: $r_{t+1}=\alpha_{i}+\varepsilon_{t+1}$; Equation 1: $r_{t+1}=\alpha_{i}+\beta_{i} x_{i, t}+\varepsilon_{t+1} ; p$-value corresponds to the test of i.i.d. residuals based on the $z$-statistic of the BDS test.

\footnotetext{
${ }^{4}$ As suggested by an anonymous referee, we conducted various tests of heteroscedasticity on the residuals of the predictive regression model. However, we could not reject the null of homoscedasticity. Complete details of these results are available upon request from the authors.
} 
In any event, for the sake of completeness and comparability, we also present the forecasting results from the linear predictive regression in Table 3, besides the same from the quantile predictive regressions over $\tau=0.10,0.20,0.30 \ldots .0 .90$. The entries in the table reports the ratio of the root mean square forecast errors (RMSFEs) ${ }^{5}$ of equation (1) relative the historical average (i.e., $\hat{r}_{i, t+1}=\hat{\alpha}_{i}$ ), and the same for equation (2) relative to the prevailing quantile model $\left(\hat{r}_{i, t+1}(\tau)=\alpha_{i}{ }^{(\tau)}\right)$. If the ratio is less than one, then the model with the predictor outperforms the model without it. It is also important to test whether the superior performance of the model with the DRCA, if it holds, is statistically different from the appropriate benchmark. Given the models nests its corresponding benchmark, we use the MSE-F test statistic of McCracken (2007), to check if, in cases where the ratio is less than one, whether the model with changes in real current account balance outperforms the one without in a significant fashion.

As can be seen from Table 3, for the linear predictive regressions, the model with DRCA fails to beats the forecasting performance of the benchmark. However, given the evidence non-normality and nonlinearity, these results cannot be relied on. Thus we move on to the quantile predictive regression model. As we observe from Table 3 again, the quantile regression model with DRCA outperforms the prevailing quantile benchmark significantly at one percent level for $\tau \in[0.10,0.30]$, i.e., around the lower-end of the conditional distribution of the equity premium. ${ }^{6}$ However, DRCA fails to provide any

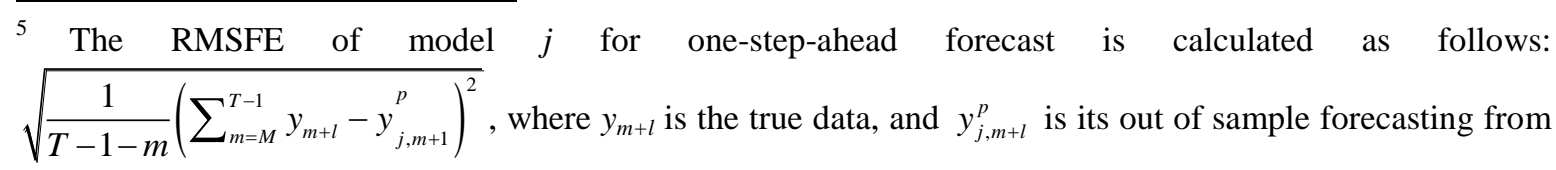
model $j ; m$ is the initial forecast origin; and $T$ is the sample size.

${ }^{6}$ Qualitatively similar, though slightly weaker, forecasting gains were obtained under the quantile predictive regression model untill the quantile range of 0.30 , when we used the changes in nominal current account balance instead of the real values of the same. The linear (conditional mean-based) predictive regression model continued to show no evidence of predictability using changes in nominal current account balance. In addition to using the nominal current account, our results were qualitatively same when we used the S\&P500 instead of the CRSP-VW index to compute the excess returns, i.e., forecasting gains were only concentrated around the lower quantiles, with the conditional mean-based model producing no gains. Using an alternative metric for the measure of forecasting performance, namely the Mean Absolute Percentage Forecast Errors 
forecastability beyond the quantile value of 0.30 . In sum, unlike as suggested by the linear (mean-based) predictive regression model, the quantile regression model shows that DRCA contains significant out-of-sample information especially when the stock market is performing poorly, but not when the market is in normal to bullish modes.

This result seems to be intuitive in the sense that, when the markets are performing average to well, that is performing around the median and above of the conditional distribution of the equity premium, the equity premium is inherently a random-walk and hence, no information, from a predictor, in our case the DRCA, can play a role in forecasting the equity premium. However, when the stock market is down, i.e., in the lower-end of the conditional distribution, information contained in the DRCA can be pivotal in forecasting stock returns. To put it alternatively, our results seem to hint towards the fact that the current account has information for predictability of the equity premium when the market is not doing well because in those states investors are looking for more macroeconomic information, while when the markets are booming there is more herding into the markets and less interest in searching out for relevant marco-economic information on behalf of the market agents.

As suggested by an anonymous referee, and in line with the current literature on forecasting excess returns (see for example, Rapach and Zhou (2013)), we also analysed the equity premium forecasts with profit- or utility-based metric, which provide more direct measures of the value of forecasts to economic agents. A leading utility-based metric for analysing equity premium forecasts is the average utility gain for a mean-variance investor. The first step is to compute the average utility for a mean-variance investor with relative risk

(MAPFE), also produced qualitatively similar results as that obtained from the relative RMSFE. However, we also observed a forecasting gain at the quantile level of 0.90 . Finally, the significance of the MSE-F test statistic was also confirmed by the $E N C-N E W$ statistic of Clark and McCracken (2001). Complete details of all these results are available upon request from the authors. 
aversion $\theta^{7}$ who allocates his portfolio between stocks and risk-free bills based on the equity premium predictive regression forecasts. This requires the investor to forecast the variance of the equity premium. Following Campbell and Thompson (2007) and Rapach and Zhou (2013), we assume that the investor allocates the following share of his portfolio to equities during $t+1$

$a_{i, t}=\frac{1}{\theta}\left(\frac{\hat{r}_{i . t+1}}{\hat{\sigma}_{t+1}^{2}}\right)$

where $\widehat{\sigma}_{t+1}^{2}$ is a forecast of the variance of the equity premium. The average utility level realised by the investor over the out-of-sample period is given by:

$\hat{v}_{i}=\hat{\mu}_{i}-0.5 \theta \hat{\sigma}_{i}^{2}$

where $\hat{\mu}_{i}$ and $\widehat{\sigma}_{i}^{2}$ are the sample mean and variance of the portfolio formed on the basis of $\widehat{r}_{i, t+1}$ and $\widehat{\sigma}_{t+1}^{2}$ over the out-of-sample forecast evaluation period. If the investor instead relies on the benchmark model of the equity premium, he allocates the portfolio share as:

$a_{0, t}=\frac{1}{\theta}\left(\frac{\bar{r}_{t+1}}{\hat{\sigma}_{t+1}^{2}}\right)$

to equity during $t+1$ and he will realise an average utility level of

$\hat{v}_{0}=\hat{\mu}_{0}-0.5 \theta \hat{\sigma}_{0}^{2}$

where $\hat{\mu}_{0}$ and $\widehat{\sigma}_{0}^{2}$ are the sample mean and variance over the out-of-sample period formed on the basis of $\bar{r}_{t+1}$ and $\widehat{\sigma}_{t+1}^{2}$. The difference between equation (4) and (6) represents the utility gain accruing to using the predictive regression forecast of the equity premium in

\footnotetext{
${ }^{7}$ Following Rapach and Zhou (2012), we report the utility gains for $\theta=3$ since the results are qualitatively similar for other reasonable $\gamma$ values.
} 
place of the benchmark forecast in the asset allocation decision. The utility gain is basically the portfolio management fee that an investor is willing to pay to have access to the additional information available in a predictive regression model relative to the information in the benchmark. As can be seen from Table 3, there are positive utility (economic) gains to have at quantiles 0.10 to 0.30 , and even quantile 0.40 , even when there are no statistical gains at this quantile.

Further, based on the suggestion of an anonymous referee, we also conducted the analysis for the Euro Area, Japan and the UK. Note, given data availability the periods studied are: Euro Area: 1997:Q2-2012:Q4; Japan: 1985:Q2-2013:Q3; UK: 1960:Q2-2014:Q1. Again, using the Bai and Perron (2003) tests of structural breaks, the out-of-sample periods started in 200:Q4 for the Euro Area, 1990:Q1 for Japan, and 1975:Q1 for the UK. ${ }^{8}$ While data on the nominal current account in US dollars (converted to real current account by dividing with the US CPI) was derived from the FRED database of Federal Reserve Bank of St. Louis, data on stock prices and the risk-free rates to compute the excess returns, were obtained from the Macroeconomic Indicators database of the OECD. The results have been reported in the Appendix of the paper (Table A1), given that the focus of the paper is the US economy. We observe that the conditional mean-based model fails to pick up out-ofsample forecasting for the Euro Area and the UK, but does so for Japan. However as with the US, the quantile predictive regression is capable of detecting predictability at for certain quantiles: Euro Area over quantiles 0.10 to 0.60 ; Japan over 0.10 to 0.50 ; and for the UK over the entire conditional distribution. Our results again highlight the importance of using a quantile predictive regression approach especially for the Euro Area and the UK, while

\footnotetext{
${ }^{8}$ The break dates obtained were as follows: Euro Area: 2000:Q4, 2003:Q2, 2007:Q3; Japan: 1990:Q1, 1995:Q3, 2000:Q3, 2004:Q3, and 2009:Q2; and the UK: 1975:Q1, 1995:Q2, and 2003:Q2.
} 
For Japan, it is still helpful in showing that the predictability is concentrated between the lower-end to the average phases of the market.

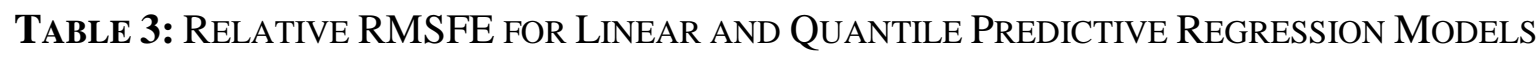

\begin{tabular}{|c|c|c|}
\hline Quantile Regression $(\tau)$ & $\begin{array}{c}\mathrm{RMSFE}_{m} / \\
\mathrm{RMSFE}_{b}\end{array}$ & $\begin{array}{c}\text { Utility Gain } \\
\text { (Annualized \%) }\end{array}$ \\
\hline 0.1 & $0.8400 * * *$ & 861.1541 \\
\hline 0.2 & $0.8870 * * *$ & 600.0650 \\
\hline 0.3 & $0.9272 * * *$ & 227.8583 \\
\hline 0.4 & 1.0036 & 12.5494 \\
\hline 0.5 & 1.0223 & -88.5344 \\
\hline 0.6 & 1.0167 & -162.2936 \\
\hline 0.7 & 1.0566 & -337.1264 \\
\hline 0.8 & 1.1069 & -457.4156 \\
\hline 0.9 & 1.0550 & -613.3201 \\
\hline Linear Regression & 1.0221 & -12.2115 \\
\hline
\end{tabular}

Note: ${ }^{* * *}$ indicates the $1 \%$ level of significance for the $M S E-F$ statistic of McCracken (2007), whilst $\tau$ specifies the quantile; $\mathrm{RMSFE}_{m} / \mathrm{RMSFE}_{b}$ signifies the Root Mean Square Forecast Error (MSFE) ratio of the corresponding linear $\left(r_{t+1}=\alpha_{i}+\beta_{i} x_{i, t}+\varepsilon_{t+1}\right)$ or quantile regression $\left(r_{t+1}=\alpha_{i}{ }^{(\tau)}+\beta_{i}{ }^{(\tau)} x_{i, t}+\varepsilon_{t+1}\right)$ models over the one generated by the benchmarks $\left(r_{t+1}=\alpha_{i}+\varepsilon_{t+1}\right.$ or $r_{t+1}=\alpha_{i}{ }^{(\tau)}+\varepsilon_{t+1}$, respectively); Utility gains as defined by equations (4) and (6) in the text.

\section{CONCLUSIONS}

The importance of accurate forecasting of stock returns for practitioners and academics in finance is well-recognized. However, stock return forecasting is highly challenging, since it inherently contain a sizable unpredictable component. Naturally, variety of models and predictors has been used in the literature. In this regard, Mercereau (2003a) developed a dynamic general equilibrium model to show that movements in the 
current account balance can predict stock returns. Some in-sample evidence was provided for the US in Mercereau (2003b).

Given this, and under the widely held view that predictive models require validation based on an out-of-sample forecasting exercise, we investigate whether the real current account balance could help in forecasting the quarterly S\&P500-based equity premium over an out-of-sample period of 1970:Q3 to 2014:Q4, with a corresponding in-sample period of 1947:Q2 to 1970:Q2. In this regard, we compare the forecastability of the US equity premium vis-à-vis the changes in the real current account balance using linear and quantile predictive regression models, with the latter capturing the nonlinearity in this relationship significant statistical evidence of which we detect in the data. The linear predictive regression model, traditionally used in the literature, with changes in real current account balance fails to outperform the benchmark model of the historical average of equity premium. However, in the presence of nonlinearity and non-normality, the linear model is found to be misspecified, and hence, its results unreliable. When we use a quantile predictive regression model, we observe that the changes in real current account balance contain significant out-of-sample information around the lower-end of the conditional distribution of the equity premium, but not when the market behavior ranges between normal to bullish, i.e., around the median to the upper quantiles. Our results, thus highlights the importance of studying the entire distribution, rather than performing just conditional mean-based analysis, which might be misleading as it is likely to miss important information contained for certain parts of the distribution of the US equity premium. As part of future research, it would be interesting to conduct a Dynamic Model Averaging (DMA) analysis on the quantile predictive regression model, which will simultaneously include other predictors used in the literature as well (see for example, Goyal and Welch (2006)). The DMA will allow us to obtain time-varying information of the importance of 
various predictors over the out-of-sample period (based on posterior inclusion probabilities) at various parts of the conditional distribution, controlling for issues of data mining - a major concern for predictive regression models with multiple predictors.

\section{REFERENCES}

Aye, G. C., Deale, F. W., and Gupta, R. Forthcoming. Does Debt Ceiling and Government Shutdown Help in Forecasting the US Equity Risk Premium? Panoeconomicus.

Bai, J., and Perron, P. 2003. Computation and analysis of multiple structural change models. Journal of Applied Econometrics 18(1), 1-22.

Bekiros, S., and Gupta, R. 2015. Predicting stock returns and volatility using consumptionaggregate wealth ratios: A nonlinear approach. Economics Letters 131, 83-85.

Bekiros, S., Gupta, R., and Kyei, C. (Forthcoming a). On Economic Uncertainty, Stock Market Predictability and Nonlinear Spillover Effects. North American Journal of Economics and Finance.

Bekiros, S., Gupta, R., and Majumdar, A. (Forthcoming b). Incorporating Economic Policy Uncertainty in US Equity Premium Models: A Nonlinear Predictability Analysis. Finance Research Letters.

Brock, W. A., Scheinkman, J. A., Dechert, W. D., and LeBaron, B. 1996. A test for independence based on the correlation dimension. Econometric reviews 15(3), 197235.

Campbell, J.Y. (2008). Viewpoint: Estimating the Equity Premium. Canadian Journal of Economics 41, 1-21.

Campbell, J.Y. and Thompson, S.B. (2008). Predicting the Equity Premium Out of Sample: Can Anything Beat the Historical Average? Review of Financial Studies, 21(4), $1509-1531$ 
Cenesizoglu, T., and Timmermann. A., (2008). Is the distribution of stock returns predictable? Available online at SSRN http://ssrn.com/abstract=1107185.

Cenesizoglu, T., and Timmermann. A., (2012). Do Return Prediction Models Add Economic Value? Journal of Banking and Finance, 36 (11), 2974-2987.

Clark, T.E., and McCracken, M. W. (2001). Test of Equal Forecast Accuracy and Encompassing for Nested Models, Journal of Econometrics, 105 (1), 85-110.

Goyal, A., and Welch, I. 2008. A Comprehensive Look at the Empirical Performance of Equity Premium Prediction, Review of Financial Studies, 21(4), pages 1455-1508.

Koenker, R., and Bassett Jr, G. 1978. Regression quantiles. Econometrica 46, 33-50.

McCracken, M. W. 2007. Asymptotics for out of sample tests of Granger causality. Journal of Econometrics 140(2), 719-752.

Meligkotsidou, L., Panopoulou, E., Vrontos, I.D., and Vrontos, S.D. (2014). A Quantile Regression Approach to Equity Premium Prediction. Journal of Forecasting, 33 (7), $558-576$.

Mercereau, B., 2003a. The Role of Stock Markets in Current Account Dynamics: a Time Series Approach. The B.E. Journal of Macroeconomics 3 (1), $1\{30$.

Mercereau, B., 2003b. The Role of Stock Markets in Current Account Dynamics: Evidence from the United States. IMF Working Papers 03/108, International Monetary Fund.

Rapach, D., and Zhou, G. 2013. Forecasting Stock Returns, in Handbook of Economic Forecasting, Volume 2A, Graham Elliott and Allan Timmermann (Eds.) Amsterdam: Elsevier, 328-383. 


\section{APPENDIX}

Table A1: Relative RMSFE for Linear and Quantile Predictive Regression MOdELS (EURO AREA, JAPAN, UK)

\begin{tabular}{|c|c|c|c|}
\hline Quantile Regression $(\tau)$ & $\begin{array}{c}\text { Euro Area: } \\
\mathrm{RMSFE}_{m} / \\
\mathrm{RMSFE}_{b}\end{array}$ & $\begin{array}{c}\text { Japan: } \\
\mathrm{RMSFE}_{m} / \\
\mathrm{RMSFE}_{b}\end{array}$ & $\begin{array}{c}\text { UK: } \\
\mathrm{RMSFE}_{m} / \\
\mathrm{RMSFE}_{b}\end{array}$ \\
\hline 0.1 & $0.7522^{* * *}$ & $0.9454 * * *$ & $0.9975 * *$ \\
\hline 0.2 & $0.7676^{* * *}$ & $0.8334 * * *$ & $0.9073 * * *$ \\
\hline 0.3 & $0.9011^{* * *}$ & $0.8537 * * *$ & $0.8989 * * *$ \\
\hline 0.4 & $0.9193 * * *$ & $0.9119 * * *$ & $0.9550^{* * *}$ \\
\hline 0.5 & $0.8972 * * *$ & $0.9889 * * *$ & $0.9597 * * *$ \\
\hline 0.6 & $0.9310^{* * *}$ & 1.0221 & $0.9507 * * *$ \\
\hline 0.7 & 1.1223 & 1.0288 & $0.9055^{* * *}$ \\
\hline 0.8 & 1.3104 & 1.1128 & $0.9380 * * *$ \\
\hline 0.9 & 1.3890 & 1.3487 & $0.8938 * * *$ \\
\hline Linear Regression & 1.0203 & $0.9849 * *$ & 1.0142 \\
\hline
\end{tabular}

Note: See Notes to Table $3 .^{* *}$ indicates the $5 \%$ level of significance for the MSE-F statistic of McCracken (2007). 